\title{
QUISTE PROSTÁTICO DE LÍNEA MEDIA EN PACIENTE TRASPLANTADO RENAL
}

\author{
O. GONZÁLEZ GARCÍA, J. SOTO VILLALBA, M. ROMERO TENORIO, \\ J.M. FARIÑAS VARO, J. LÓPEZ CÓZAR, J. FLORES GINÉS
}

Servicio de Urología. Hospital Universitario Puerta del Mar. Cádiz.

Actas Urol Esp. 28 (6): 452-454, 2004

\section{RESUMEN}

QUISTE PROSTÁTICO DE LÍNEA MEDIA EN PACIENTE TRASPLANTADO RENAL

Las lesiones quísticas localizadas en la línea media de la próstata son poco frecuentes y se consideran alteraciones congénitas. Se acepta generalmente que el tratamiento activo se realiza sólo a los pacientes sintomáticos, haciendo observación a los asintomáticos. El tratamiento activo más común es la cirugía mínimamente invasiva por medio de punción-aspiración o la decorticación endoscópica.

Aportamos un caso de un paciente inmunosuprimido por un trasplante renal, circunstancia que nos hizo optar por el tratamiento activo con punción aspiración del mismo, a pesar de ser asintomático.

PALABRAS CLAVE: Quiste prostático. Observación. Quiste de la línea media. Trasplante renal.

\section{ABSTRACT}

PROSTATE CYST AT THE MIDLINE IN A PATIENT WITH A RENAL TRANSPLANTATION

Cystic lesions at the midline of the prostate are uncommon and they are considered as congenital disorders. Is generally accepted that active treatment should be instituted only in symptomatic patients and watchful waiting in asymptomatic ones. The most common active treatment is minimally invasive surgery by puncture-aspiration or endoscopic unroofing.

We are attaching an inmunosupressed patient because of a renal transplantation in which we provided an active treatment by puncture-aspiration of the cystic lesion in spite of being asymptomatic. KEY WORDS: Prostatic cyst. Watchful waiting. Midline cyst. Renal transplantation.

$\mathrm{L}^{\mathrm{a}}$ as lesiones quísticas ${ }^{1-4}$ de la próstata son hallazgos muy habituales y se pueden dividir en laterales y mediales, o de linea media. Las primeras son las más frecuentes y suelen aparecer en el contexto de la hiperplasia benigna de próstata y de procesos inflamatorios de cualquier etiología. Los quistes de linea media son raros y se los considera como de origen congénito. Estos los hay de dos tipos ${ }^{5,6}$ : los quistes müllerianos, derivados de restos de conducto paramesonéfrico, y los quistes de utrículo, probablemente causado por dilatación de utrículo prostático. En ambos casos la morfología y el comportamiento clínico son similares a pesar de tan distinto origen $\mathrm{y}$ tan discutido.

En general se acepta que el tratamiento ${ }^{1}$ activo se debe reservar para los pacientes sintomáticos y la observación para los asintomáticos.

Aportamos en este trabajo un caso de un paciente asintomático en el que, a pesar de ello, optamos por el tratamiento por punciónaspiración debido a las condiciones del paciente, su inmunosupresión por ser trasplantado renal. 


\section{CASO CLÍNICO}

El paciente es un varón de cuarenta y tres años de edad, sin antecedentes de diabetes, hipertensión ni reacciones alérgicas a fármacos. Así mismo no presenta malformaciones congénitas de ningún tipo ni antecedentes familiares de interés.

Hace seis años debuta con un síndrome nefrótico leve de progresiva evolución a insuficiencia renal. Se realizan estudios serológicos, negativos todos, $\mathrm{y}$ tras el estudio inmunohistoquímico y biopsia renal se diagnostica de glomerulonefritis membrano-proliferativa. El paciente sigue un curso evolutivo pobre hacia una insuficiencia renal severa que hace que tenga que comenzar con programa de hemodiálisis. Hace un año recibe trasplante renal de donante cadáver con buena evolución clínica y bioquímica, con creatininemias mantenidas alrededor de $2,0 \mathrm{mg} / \mathrm{dl}$.

En el curso de una ecografia de control posttrasplante se visualiza la próstata por vía abdominal, observándose una formación quística redondeada en posterior de la próstata, de $21 \mathrm{x}$ $23 \mathrm{~mm}$, anecoica en su interior y sin cápsula aparente. Ante este hallazgo se remite a Nuestro Servicio para valoración urológica. Se trata de un hallazgo incidental en un paciente asintomático. Decidimos realizar una ecografía transrectal de la próstata donde comprobamos la existencia de la formación quística de esas dimensiones a nivel de la Zona Central de la próstata, a nivel de la línea media, que no parece afectar a los conductos eyaculadores (Fig. 1).

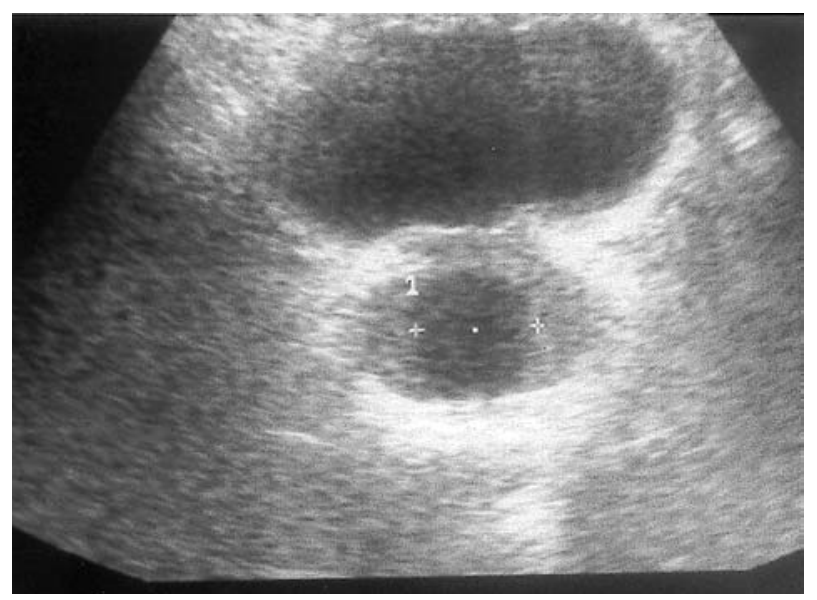

FIGURA 1
Dada la situación de inmunosupresión del paciente por su trasplante $\mathrm{y}$, ante la posibilidad de tratarse de un quiste de utrículo con posible comunicación a la uretra, decidimos hacer tratamiento activo a pesar de ser asintomático. Con la profilaxis antibiótica para los procedimientos transrectales de próstata, realizamos punciónaspiración del quiste con intención diagnóstica y terapéutica, tomando la muestra para citología y cultivo, y comprobando el vaciado total del quiste. Nos encontramos con un fluido pardo, sin restos macroscópicos de coágulos, que en la citología no presenta células sospechosas de malignidad ni semen. Los cultivos para aerobios y anaerobios han resultado negativos.

\section{DISCUSIÓN}

Los quistes de línea media ${ }^{4}$ de la próstata son hallazgos poco frecuentes, situándose a nivel posterior de la uretra, sin interesarla, proximal a la desembocadura de los conductos eyaculadores. Se consideran todos como de origen congénito, aunque su verdadera patogenia ha sido objeto de acaloradas discusiones. En general, se acepta hoy que son de dos tipos ${ }^{5,6}$ : los derivados de restos de conducto de Müller o paramesonéfrico, llamados quistes müllerianos; y los derivados de la dilatación de utrículo prostático, los quistes de utrículo.

Con gran frecuencia son asintomáticos ${ }^{1} \mathrm{y}$ su hallazgo es incidental en el curso de pruebas de imagen para estudio de otros procesos, principalmente durante la ecografía abdominal o transrectal. También pueden aparecer síntomas como la hematuria o los derivados de la obstrucción de la uretra o de los conductos eyaculadores ${ }^{7}$, o de la infección del quiste.

El diagnóstico se realiza por imagen ${ }^{1,3,8,9}$ y basta con la ecografía vía abdominal, en la que se ve la formación anecoica no encapsulada a nivel de zona central de próstata, proximal a veru montanum. La TAC aporta una imagen parecida de baja densidad, en un rango entre 10 y $25 \mathrm{UH}$, sin refuerzo alrededor y exactamente en la línea media (Fig. 2).

Para plantear el manejo no va a aportar nada la diferenciación del origen del quiste, y por tanto del tipo, ya que su comportamiento clínico va a ser el mismo. A pesar de ello y a nivel académico, 


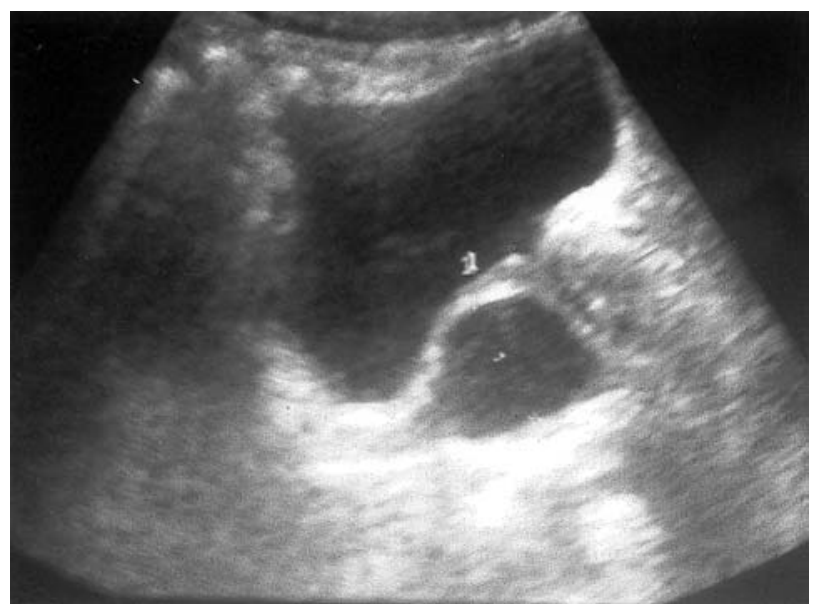

FIGURA 2

cuando podamos disponer de la histología por prostatectomía o decorticación del quiste en su caso y por ser necesario, podemos sospechar que sea quiste de utrículo $^{5}$ cuando haya epitelio endodérmico y mesodérmico, cuando encontremos comunicación a uretra o cuando haya malformaciones asociadas como hipospadias o genitales ambiguos. En los müllerianos sólo aparece epitelio mesodérmico y no asocia comunicación a uretra ni malformaciones.

La indicación de tratamiento debe ser reservada sólo para los casos sintomáticos. La técnica a usar no está claramente establecida aunque, dados los malos resultados de la cirugía abierta y por el riesgo quirúrgico de la misma, se prefiere la punciónaspiración ${ }^{10}$ por vía transperineal o transrectal, o la decorticación endoscópica de la lesión cuando ésta protruya a vejiga. En ambos casos se han publicado buenos resultados a largo plazo.

En los pacientes asintomáticos está indicada la observación y seguimiento a largo plazo con una cadencia no establecida, ya que se ha descrito un riesgo de malignización de alrededor de un $3 \%^{11,12}$. Si bien la anterior indicación es la aceptada, en nuestro caso, aparte de la existencia de síntomas, valoramos las circunstancias de salud del paciente y la incidencia psicológica del proceso en el mismo. En cuanto a lo primero, se trata de un paciente inmunodeprimido y con alto riesgo de infección y sepsis; en cuanto a lo segundo, el paciente prefirió el tratamiento activo ya que le causaría una gran ansiedad la mera observación de la formación quística. Sobre la base de estas circunstancias optamos por la punción- aspiración del quiste y su seguimiento semestral, ampliando las ecografias de control nefrológico de su trasplante para control prostático.

En conclusión, pensamos que se trata de una patología cuyo diagnóstico, que hoy es raro, se va a hacer cada vez más frecuente, de forma incidental, por la gran difusión de las pruebas de imagen abdomino-pélvicas y la extensión de la ecografía transrectal de la próstata. Así mismo, estamos de acuerdo en la abstención de tratamiento y la observación en los pacientes asintomáticos, aunque pensamos que además se deben valorar también las circunstancias clínicas y psicológicas del mismo.

\section{REFERENCIAS}

1. TORNERO RUIZ J, PONCE DE LEÓN ROCA J, PIERAS AYALA E et al.: Quistes prostáticos de la línea media: manejo y seguimiento. Arch Esp Urol 2001; 54: 205-209.

2. GARCÍA MEDIERO JM, NÚÑEZ MORA C, PÉREZ MIES B et al.: Quistes mesoteliales en urología; un problema diagnóstico y terapéutico. Arch Esp Urol 2002; 55 (1): 73-76.

3. NGHIEM HT, KELLMAN GM, SANDBERG SA et al.: Cystic lesions of the prostate. Radiographics 1990; 10: $635-650$.

4. ZHU JP, MEYHOFF HH.: Prostatic cyst. Scand $J$ Urol Nephrol; 29: 345-349.

5. HARUAKI K, HITSUKI K, TOSHITAKA $M$ et al.: Histopathological study of the Müllerian duct remnant: clarification of disease categories and terminology. J Urol 2002; 167: 133-136.

6. YASUMOTO R, KAWANO M, TSUJINO K.: Is a cystic lesion located at the midline of the prostate a Müllerian duct cyst?. Eur Urol 1997; 31: 187-189.

7. VAN POPPEL H, VEREECKEN R, DE GEETER P et al. Hemospermia owing to utricular cyst: embryological summary and surgical review. J Urol 1983; 129: 608-609.

8. SCHWARTZ JM, BOSNIAK MA, HULNIK DH et al. Computed tomography of midline cysts of the prostate. J Comput Assist Tomogr 1988; 12 (2): 215-218.

9. RITCHEY ML, BENSON RC, KRAMER SA et al. Management of Müllerian duct remnants in the male. J Urol 1988; 140: 795-799.

10. FISCHELOVITCH D, MEIRAZ D, LAZEBNIK J.: Cysts of the prostate. BJU 1975; 47: 687-689.

11. NOVAK RW, RAINES RB, SOLLEE AN.: Clear cell carcinoma in a Müllerian duct cyst. Am J Clin Pathol 1981; 76: 339-341.

12. SZEMES GC, RUBIN DJ.: Squamous cell carcinoma in a Müllerian duct cyst. J Urol 1968; 100: 40-43.

Dr. O. González García

Serv. de Urología. Hospital Univ. Puerta del Mar

Avda. Ana de Viya, 21

11009 Cádiz

(Trabajo recibido el 2 septiembre de 2003) 